\title{
Diagnostic Accuracy of Elevated Serum Carcinoembryonic Antigen for Recurrence in Postoperative Stage II Colorectal Cancer Patients: Comparison With Stage III
}

\author{
Ho Seung Kim, Min Ro Lee \\ Research Institute of Clinical Medicine, Chonbuk National University Medical School, Jeonju, Korea
}

Purpose: Elevated levels of serum carcinoembryonic antigen (CEA) following a curative resection of colorectal cancer (CRC) indicate recurrence; however, the levels of CEA may be elevated above the normal limit without recurrence. The aim of this study is to analyze the diagnostic accuracy of elevated serum CEA for predicting recurrence in postoperative stage II and stage III CRC patients.

Methods: A total of 336 stage II and stage III CRC patients who underwent a curative resection between January 2005 and October 2009 were enrolled. Sensitivities, specificities, positive predictive values (PPVs), negative predictive values (NPVs), likelihood ratios and post-test probabilities of recurrence associated with elevated CEA were analyzed and compared.

Results: The median follow-up duration was 45 months (36 to 134 months). Twenty-seven of 189 stage II patients (14.3\%) and 52 of 147 stage III patients (35.4\%) developed recurrence during the follow-up period. Sensitivities, specificities, PPVs, and NPVs of elevated CEA were $37.0 \%, 91.4 \%, 41.7 \%$, and $89.7 \%$, respectively, in stage II patients and $46.2 \%$, $90.5 \%, 72.7 \%$, and $75.4 \%$ in stage III patients. Post-test probabilities of recurrence associated with elevated CEA were $41.8 \%$ in stage II patients and $71.9 \%$ in stage III patients.

Conclusion: The predictive performance of the probability of recurrence associated with elevated serum CEA after a curative resection in stage II CRC patients is lower than that in stage III CRC patients.

Keywords: Carcinoembryonic antigen; Tumor marker; Colorectal neoplasms; Recurrence; Accuracy

\section{INTRODUCTION}

Recurrence after a curative resection of colorectal cancer (CRC) occurs in $30-40 \%$ of patients. However, resections of limited metastatic disease may result in a potential cure [1]. Therefore, early detection of recurrence is of paramount importance to identify

Received: May 8, 2013 - Accepted: July 24, 2013

Correspondence to: Min Ro Lee, M.D.

Research Institute of Clinical Medicine, Chonbuk National University

Medical School, 567 Baekje-daero, Deokjin-gu, Jeonju 561-756, Korea

Tel: +82-63-250-1570, Fax: +82-63-271-6197

E-mail:gsminro@chonbuk.ac.kr

(c) 2013 The Korean Society of Coloproctology

This is an open-access article distributed under the terms of the Creative Commons Attribution NonCommercial License (http://creativecommons.org/licenses/by-nc/3.0) which permits unrestricted noncommercial use, distribution, and reproduction in any medium, provided the original work is properly cited. patients who may benefit from salvage surgery. Carcinoembryonic antigen (CEA) has been most commonly used as a tumor marker to detect early recurrence amenable to curative surgery. In spite of its widespread use and cost effectiveness [2], little is known about the accuracy and the efficacy of CEA monitoring [3-5]. An elevated level of serum CEA following a curative resection of the CRC may indicate recurrence; however, the levels of CEA may be elevated above the normal limit without recurrence. Also, whether the accuracy of CEA monitoring in stage II is comparable to that in stage III is not known.

The likelihood ratio and the post-test probability have been recently reported to be superior to the sensitivity and the specificity in comparing the accuracy of a test because they are less influenced by the morbidity rate [6]. Here, we analyze the diagnostic accuracy of elevated serum CEA in postoperative stage II and stage III CRC patients by using the likelihood ratios and the post- 
test probabilities, as well as the sensitivities, specificities, positive predictive values (PPVs) and negative predictive values (NPVs).

\section{METHODS}

Patients who underwent a radical resection for CRC between January 2005 and October 2009 at Chonbuk National University Hospital were enrolled. Patients with stage 0, I or IV cancer, insufficient follow-up (less than three years), abnormal CEA in the first measurement after surgery (checked within three months after surgery), history of other cancers and/or history of preoperative concurrent chemoradiation therapy were excluded. Finally, 336 patients with stage II $(n=189)$ or III $(n=147)$ CRC were included.

CEA was measured using an immunoassay method (ADIVA Centaur XP immunoassay system, Siemen AG, Erlangen, Germany). CEA elevation was defined as levels above $5 \mathrm{ng} / \mathrm{mL}$. All patients underwent routine serum CEA assays and radiologic examination for 5 years. CEA levels were assayed with a 3-month interval for the first 2 years and every 6 months thereafter. Chest computed tomography (CT) and abdomino-pelvic CT were performed with a 6-month interval for the first 2 years and every 1 year thereafter. The diagnosis of a tumor recurrence was confirmed by biopsy and radiologic evidence. We also obtained sensitivities, specificities, PPVs, and NPVs.

Determination of elevated CEA and recurrence was done each postoperative year (POY): POY was defined as the period within 1 year after surgery, POY2 as $\geq 1$ but $<2$ years, POY 3 as $\geq 2$ but $<3$ years, and POY 4 as $\geq 3$ years. The recurrence rate in any given year was obtained by calculating the number of recurrences divided by the number of patients followed for that year.

The sensitivity was defined as the fraction of all diseased patients with a positive test. The specificity was defined as the fraction of all healthy patients with a negative test. The likelihood ratio for a positive test was defined as the ratio of the fraction of true positives to the fraction of false positives (sensitivity/[1-specificity]). The likelihood ratio for a negative test was defined as the fraction of false negatives to the true negatives ([1-sensitivity]/specificity).

The pretest probability was defined as the probability that a patient suffered from recurrence. The post-test probability was defined as the probability of recurrent disease in a patient with a

Table 1. Statistical analysis

\begin{tabular}{l}
\hline Statistical analysis \\
\hline Sensitivity = true positive/(true positive + false negative) \\
Specificity = true negative/(true negative + false positive) \\
Likelihood ratio (test positive) $=$ sensitivity/(1-specificity) \\
Likelihood ratio (test negative) $=(1$-sensitivity)/specificity \\
Pretest odds = pretest probability/(1-pretest probability) \\
Post-test odds = likelihood ratio $\times$ pretest odds \\
Post-test probability = post-test odds/(1 + post-test odds) \\
\hline
\end{tabular}

certain test result for CEA. Thus, the likelihood ratio positive $(\mathrm{LR}+)$ is a measurement of the increase in the probability of disease according to a certain test result. $\mathrm{LR}+>10$ and $\mathrm{LR}-<0.1$ were considered to be excellent test results ( $\mathrm{LR}+>10$ : a positive test is ten times more likely to occur in a patient with recurrence; LR$<0.1$ : a false negative test result will occur in less than one out of ten patients with a negative test) (Table 1 ).

\section{RESULTS}

\section{Patient characteristics}

The median follow-up duration was 45 months (36 to 134 months). Of the 336 patients, 189 (56.3\%) were stage II, and 147 (43.7\%) were stage III. There was no difference between the two groups, stage II and stage III groups, except for the recurrence rate (Table 2).

\section{Pretest probability}

In the stage II group, recurrence developed in 27 patients (14.3\%). The yearly recurrence rates of POY1, POY2, POY3, and POY4 were $4.2 \%(8 / 189)$, $5.5 \%(10 / 181), 1.8 \%(3 / 171)$, and $3.6 \%(6 / 168)$ respectively. On the other hand, in the stage III group, recurrence

Table 2. Clinicopathologic findings of patients according to stage

\begin{tabular}{|c|c|c|c|}
\hline Variable & Stage II $(n=189)$ & Stage III $(n=147)$ & P-value \\
\hline Age (yr) & $60.4 \pm 11.1(33-83)$ & $60.4 \pm 10.7(29-81)$ & 0.842 \\
\hline Gender (M/F) & $122 / 67$ & $82 / 65$ & 0.103 \\
\hline Location & & & 0.484 \\
\hline Colon & 129 & 95 & \\
\hline Rectum & 60 & 52 & \\
\hline Depth & & & $<0.001$ \\
\hline $\mathrm{T} 1$ & 0 & 2 & \\
\hline T2 & 0 & 11 & \\
\hline T3 & 185 & 127 & \\
\hline $\mathrm{T} 4$ & 4 & 7 & \\
\hline pN stage & & & $<0.001$ \\
\hline NO & 189 & 0 & \\
\hline N1 & 0 & 102 & \\
\hline N2 & 0 & 45 & \\
\hline Size (cm) & $5.4 \pm 2.1(1.2-13.7)$ & $4.7 \pm 1.7(1.3-9.1)$ & 0.590 \\
\hline $\begin{array}{l}\text { Preoperative CEA } \\
\text { (ng/mL) }\end{array}$ & & & 0.184 \\
\hline$\leq 5$ & $141(74.6)$ & $100(68.0)$ & \\
\hline$>5$ & $48(25.4)$ & $47(32.0)$ & \\
\hline $\begin{array}{l}\text { Preoperative CEA } \\
(\mathrm{ng} / \mathrm{mL})\end{array}$ & $8.8 \pm 26(0.5-301.9)$ & $8.1 \pm 17.5(0.7-169.2)$ & 0.056 \\
\hline Recurrence & 27 (14.3) & $52(35.4)$ & $<0.001$ \\
\hline
\end{tabular}

Values are presented as mean \pm standard deviation (range) or number (\%). CEA, carcinoembryonic antigen. 
developed in 52 patients (35.4\%). The yearly recurrence rates were $19.0 \%$ (28/147), 13.4\% (16/119), 4.9\% (5/103), and 3.1\% (3/98), respectively (Table 3 ). These rates were regarded as the pretest probabilities in this study.

\section{Sensitivity, specificity, PPV, NPV in stage II and III}

The overall sensitivity, specificity, PPV, and NPV of the $336 \mathrm{pa}-$ tients investigated were $43.0 \%, 91.1 \%, 59.6 \%$, and $83.9 \%$, respectively. In stage II patients, the results were $37.0 \%, 91.4 \%, 41.7 \%$, and $89.7 \%$, respectively, and in stage III patients, they were $46.2 \%$, $90.5 \%, 72.7 \%$, and $75.4 \%$ (Table 4 ).

\section{Sensitivity, specificity, and LR in stages II and III each year} In stage II patients with recurrence, CEA elevation was observed in $4,4,1$, and 1 patients in POY1, POY2, POY3, and POY4, respectively. The yearly sensitivities of elevated CEA for predicting recurrence were $50.0 \%, 40.0 \%, 33.3 \%$, and $16.7 \%$, respectively. In stage II patients without recurrence, CEA was not elevated in 176, 166,166 , and 160 patients in POY1, POY2, POY3, and POY4, respectively, and the yearly specificities were $97.3 \%, 97.1 \%, 98.8 \%$, and $98.8 \%$. Likewise, in stage III patients, the yearly sensitivities

Table 3. Yearly recurrence rate after surgery according to stage

\begin{tabular}{lccccc}
\hline & \multicolumn{4}{c}{ Postoperative year } & \multirow{2}{*}{ Total } \\
\cline { 2 - 5 } & 1 & 2 & 3 & 4 & \\
\hline Stage II & $8 / 189$ & $10 / 181$ & $3 / 171$ & $6 / 168$ & $27 / 189$ \\
& $(4.2)$ & $(5.5)$ & $(1.8)$ & $(3.6)$ & $(14.3)$ \\
Stage III & $28 / 147$ & $16 / 119$ & $5 / 103$ & $3 / 98$ & $52 / 147$ \\
& $(19.0)$ & $(13.4)$ & $(4.9)$ & $(3.1)$ & $(35.4)$ \\
\hline
\end{tabular}

Values are presented as number (\%).

Table 4. Elevated serum carcinoembryonic antigen for recurrence: sensitivity, specificity, PPV, and NPV based on stage

\begin{tabular}{lccccc}
\hline Variable & No. & Sensitivity (\%) & Specificity (\%) & PPV (\%) & NPV (\%) \\
\hline Total & 336 & 43.0 & 91.1 & 59.6 & 83.9 \\
Stage II & 189 & 37.0 & 91.4 & 41.7 & 89.7 \\
Stage III & 147 & 46.2 & 90.5 & 72.7 & 75.4 \\
\hline
\end{tabular}

PPV, positive predictive value; NPV, negative predictive value. were $42.3 \%$, 56.3\%, 40.0\%, and 33.3\% in POY1, POY2, POY3, and POY4, respectively, and the yearly specificities were $95.8 \%$, $97.1 \%, 99.0 \%$, and $100.0 \%$.

Using sensitivities and specificities, we calculated positive and negative LRs. In stage II patients, the positive LRs were 17.9, 13.8, 27.8 and 13.9 in POY1, POY2, POY3, and POY4, respectively, and the negative LRs were $0.5,0.6,0.7$, and 0.8 . In stage III patients, the positive and the negative LRs were 10.1, 19.4, and 40.0 (the positive LR of POY 4 could not be calculated because the denominator was 0 ) and $0.6,0.5,0.6$, and 0.7 in POY1, POY2, POY3, and POY4, respectively. There were no significant differences in sensitivity, specificity and LR between stage II and III (Table 5).

\section{Post-test probability}

The post-test probabilities with postoperative CEA elevation were $41.8 \%$ and $71.9 \%$ in stage II and III patients, respectively. In stage II patients, the corresponding post-test probabilities in POY1, POY2, POY3, and POY4, respectively, were 44.0\%, 44.5\%, 33.8\%, and $34.2 \%$ with postoperative CEA elevation and $2.1 \%, 3.4 \%$, $1.3 \%$, and $2.9 \%$ without postoperative CEA elevation. However, in stage III patients, the corresponding post-test probabilities in

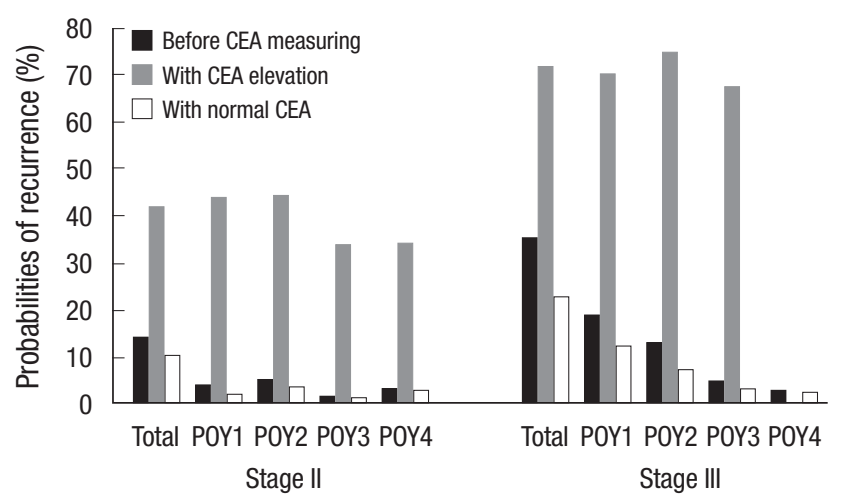

Fig. 1. Elevated serum carcinoembryonic antigen (CEA) for recurrence: probabilities of colorectal cancer recurrence according to postoperative serum CEA status (black column, before test; gray column, probability when CEA is elevated; and white column, probability when CEA is normal). Post-test probability was $41.8 \%$ in stage II patients and $71.9 \%$ in stage III patients. POY, postoperative year.

Table 5. Elevated serum CEA for recurrence: sensitivities, specificities and likelihood ratios at each year

\begin{tabular}{|c|c|c|c|c|c|c|c|c|c|c|}
\hline & \multicolumn{5}{|c|}{ POY (stage II) } & \multicolumn{5}{|c|}{ POY (stage III) } \\
\hline & Total & 1 & 2 & 3 & 4 & Total & 1 & 2 & 3 & 4 \\
\hline Sensitivitya,$\%$ (n) & $37.0(10 / 27)$ & $50.0(4 / 8)$ & $40.0(4 / 10)$ & $33.3(1 / 3)$ & $16.7(1 / 6)$ & $46.2(24 / 52)$ & $42.3(12 / 28)$ & $56.3(9 / 16)$ & $40.0(2 / 5)$ & $33.3(1 / 3)$ \\
\hline Specificity', \% (n) & $\begin{array}{c}91.4 \\
(148 / 162)\end{array}$ & $\begin{array}{c}97.2 \\
(176 / 181)\end{array}$ & $\begin{array}{c}97.1 \\
(166 / 171)\end{array}$ & $\begin{array}{c}98.8 \\
(166 / 168)\end{array}$ & $\begin{array}{c}98.8 \\
(160 / 162)\end{array}$ & $\begin{array}{c}90.5 \\
(86 / 95)\end{array}$ & $\begin{array}{c}95.8 \\
(114 / 119)\end{array}$ & $\begin{array}{c}97.1 \\
(100 / 103)\end{array}$ & $\begin{array}{c}99.0 \\
(97 / 98)\end{array}$ & $\begin{array}{c}100 \\
(95 / 95)\end{array}$ \\
\hline LR (CEA elevation) & 4.3 & 17.9 & 13.8 & 27.8 & 13.9 & 4.7 & 10.1 & 19.4 & 40.0 & - \\
\hline LR (normal CEA) & 0.7 & 0.5 & 0.6 & 0.7 & 0.8 & 0.6 & 0.6 & 0.5 & 0.6 & 0.7 \\
\hline
\end{tabular}

CEA, carcinoembryonic antigen; POY, postoperative year; LR, likelihood ratio.

aSensitivity is calculated as recurrence with tumor marker elevation/recurrence. ${ }^{\text {DS}}$ Specificity is calculated as no recurrence with normal tumor marker/no recurrence. 
POY1, POY2, POY3, and POY4, respectively, were 70.3\%, 75.0\% and $67.4 \%$ with postoperative CEA elevation and $12.3 \%, 7.2 \%$, $3.0 \%$, and $2.2 \%$ without postoperative CEA elevation (the posttest probability of POY4 with CEA elevation could not be calculated because the positive LR of POY4 was not calculated) (Fig. 1).

\section{DISCUSSION}

Recurrence after a curative resection of CRC occurs in 30-40\% of patients. However, recent chemotherapeutic agents and new biologic agents have greatly improved the survival of patients with recurrent disease. Furthermore, complete resections of limited recurrent diseases may result in a cure. Thus, early detection of recurrent disease after curative surgery is crucial to identify patients who may benefit from additional surgery [7]. CEA has been most commonly used as a tumor marker to detect early recurrence and is regarded as being more effective in detecting early evidence of recurrence than any other single entity, such as liver function tests, other tumor markers, and computed tomographic scans [810]. However, few studies have addressed the differences in the diagnostic accuracy of CEA elevation in stage II and III cancer patients.

In evidence-based medicine, the LR and the post-test probability have been used to assess the value of performing a diagnostic test [11] and have been considered to be more reliable than the sensitivity and the specificity in comparing the accuracy of tests for differences in morbidity and to be a useful measurement of the probability of obtaining a positive or negative test result [12-14]. In CRC follow-up, annual postoperative recurrence rates vary between stages II and III, indicating that the LR and the post-test probability are more suitable than the sensitivity and the specificity as accurate measures for yearly tumor-marker monitoring [15].

In the present study, no differences in sensitivity, specificity, PPV, and NPV were found between the stage II and III groups. However, the post-test probabilities with postoperative CEA elevation were found to be different, $41.8 \%$ in stage II patients and $71.9 \%$ in stage III patients. The accuracy of postoperative CEA to detect recurrence was lower in stage II patients, which could be due the low incidence of recurrence and the high false-positive rate of CEA in stage II patients [15].

Many studies have reported on the effectiveness of CEA and its diagnostic accuracy, but few studies have compared CEA elevation according to stage. CEA elevation has been reported to be diminished in value as a tumor marker in early stages [16]. However, the results were based on preoperative CEA levels according to stage, and follow-up patients after surgical treatment were not included.

Related to the postoperative diagnostic accuracy of CEA elevation after surgery, Hara et al. [15,17] also reported results similar to those of our study, with post-test probabilities of about $75 \%$ in stage III and 30\% in stage II in the first postoperative year and with efficacy drops below $50 \%$ after the first year in stage III patients, decreasing progressively year by year. In our study, the post- test probability was continually maintained at $70 \%$ in stage III.

Our study has a few limitations. Firstly, as a retrospective study, clinical factors that could affect the results were not included in the analysis as CEA can be elevated by a few benign conditions such as smoking, chronic kidney disease, chronic obstructive pulmonary disease and liver disease [18]. Secondly, we did not consider the effect of adjuvant chemotherapy. Thirdly, the diagnostic accuracy of a one-time elevation of CEA was evaluated without serial follow-up to exclude temporary elevation [19]. Nonetheless, the present study showed that in stage III patients, postoperative CEA monitoring had a high accuracy for detecting recurrence. However, in stage II patients, CEA elevation indicated recurrence with around $40 \%$ probability, which is not reliable, and clinician always expect a high possibility of false positivity.

In conclusion, the predictive performance of the probability of recurrence associated with elevated serum CEA after a curative resection in stage II CRC patients is lower than it is in stage III colorectal cancer patients.

\section{CONFLICT OF INTEREST}

No potential conflict of interest relevant to this article was reported.

\section{REFERENCES}

1. Guyot F, Faivre J, Manfredi S, Meny B, Bonithon-Kopp C, Bouvier AM. Time trends in the treatment and survival of recurrences from colorectal cancer. Ann Oncol 2005;16:756-61.

2. Rockall TA, McDonald PJ. Carcinoembryonic antigen: its value in the follow-up of patients with colorectal cancer. Int J Colorectal Dis 1999;14:73-7.

3. Korner H, Soreide K, Stokkeland PJ, Soreide JA. Diagnostic accuracy of serum-carcinoembryonic antigen in recurrent colorectal cancer: a receiver operating characteristic curve analysis. Ann Surg Oncol 2007;14:417-23.

4. Fernandes LC, Kim SB, Saad SS, Matos D. Value of carcinoembryonic antigen and cytokeratins for the detection of recurrent disease following curative resection of colorectal cancer. World J Gastroenterol 2006;12:3891-4.

5. Bhandari M, Guyatt GH. How to appraise a diagnostic test. World J Surg 2005;29:561-6.

6. Hara M, Kanemitsu Y, Hirai T, Komori K, Kato T. Negative serum carcinoembryonic antigen has insufficient accuracy for excluding recurrence from patients with Dukes $\mathrm{C}$ colorectal cancer: analysis with likelihood ratio and posttest probability in a follow-up study. Dis Colon Rectum 2008;51:1675-80.

7. Holt A, Nelson RA, Lai L. Surveillance with serial serum carcinoembryonic levels detect colorectal cancer recurrences in patients who are initial nonsecretors. Am Surg 2010;76:1100-3.

8. Rocklin MS, Senagore AJ, Talbott TM. Role of carcinoembryonic antigen and liver function tests in the detection of recurrent 
colorectal carcinoma. Dis Colon Rectum 1991;34:794-7.

9. Filella X, Molina R, Pique JM, Garcia-Valdecasas JC, Grau JJ, Novell F, et al. Use of CA 19-9 in the early detection of recurrences in colorectal cancer: comparison with CEA. Tumour Biol 1994; 15:1-6.

10. Hall NR, Finan PJ, Stephenson BM, Purves DA, Cooper EH. The role of CA-242 and CEA in surveillance following curative resection for colorectal cancer. Br J Cancer 1994;70:549-53.

11. Harrell FE Jr, Califf RM, Pryor DB, Lee KL, Rosati RA. Evaluating the yield of medical tests. JAMA 1982;247:2543-6.

12. Sackett DL, Strauss SE, Richardson WS. Evidence-based medicine: how to practice and teach EBM. 2nd ed. New York: Churchill Livingstone; 2000.

13. Akobeng AK. Understanding diagnostic tests 2: likelihood ratios, pre- and post-test probabilities and their use in clinical practice. Acta Paediatr 2007;96:487-91.

14. Davidson M. The interpretation of diagnostic test: a primer for physiotherapists. Aust J Physiother 2002;48:227-32.

15. Hara M, Sato M, Takahashi H, Takayama S, Takeyama H. Does serum carcinoembryonic antigen elevation in patients with postoperative stage II colorectal cancer indicate recurrence? Comparison with stage III. J Surg Oncol 2010;102:154-7.

16. Goldstein MJ, Mitchell EP. Carcinoembryonic antigen in the staging and follow-up of patients with colorectal cancer. Cancer Invest 2005;23:338-51.

17. Hara M, Sato M, Takahashi H, Takayama S, Takeyama H. Accuracy of monitoring serum carcinoembryonic antigen levels in postoperative stage III colorectal cancer patients is limited to only the first postoperative year. Surg Today 2011;41:1357-62.

18. Wang JY, Lu CY, Chu KS, Ma CJ, Wu DC, Tsai HL, et al. Prognostic significance of pre- and postoperative serum carcinoembryonic antigen levels in patients with colorectal cancer. Eur Surg Res 2007;39:245-50.

19. Locker GY, Hamilton S, Harris J, Jessup JM, Kemeny N, Macdonald JS, et al. ASCO 2006 update of recommendations for the use of tumor markers in gastrointestinal cancer. J Clin Oncol 2006;24: 5313-27. 